\title{
ESTIMATION OF FAST FADING CHANNEL IN IMPULSE NOISE ENVIRONMENT
}

\author{
C. Y. Fung and S. C. Chan
}

\author{
Department of Electrical and Electronic Engineering \\ The University of Hong Kong
}

\begin{abstract}
This paper studies the estimation of fast fading channel in the present of impulse noise. Fast fading channel in wireless communications system is typically modeled as autoregressive (AR) process. Least-square algorithm and Kalman filter are previously proposed for estimating the AR parameters and the channel impulse response respectively using training sequence. The performance of these algorithms, however, is very sensitive to impulse noise. In this paper, a robust Kalman filter and a robust recursive least $\mathrm{M}$-estimate algorithm are employed to jointly estimate the channel impulse response and the AR parameters of fast fading channel under impulse noise. Simulation showed that the proposed algorithms are much less sensitive to impulse noise than the conventional algorithms.
\end{abstract}

\section{INTRODUCTION}

Fast fading channel is frequently encountered in wireless communications, causing considerable performance degradation. The impulse response of the time-varying channel (TVC) can be modeled as an autoregressive (AR) process [1]. Many authors have proposed efficient algorithms for channel estimation and equalization of the TVC using Kalman filter and DecisionFeedback equalizer. In [1], a least-square (LS) method is proposed for the AR parameters estimation of the fading channel model. A coupled estimator consisting of a Kalman filter in parallel with a LS-based AR parameters estimator was proposed in [2]. Several authors also considered the estimation and equalization of MIMO channel model [3,4].

Although these algorithms work well in additive white Gaussian noise channel, their performances can be significantly degraded in impulse noise environment, which often appears in communications channel. It is because both the Kalman filter and the LS algorithm are sensitive to impulse noise, thus affecting the accuracy of the channel and $A R$ parameters estimation. As a result, the received signal cannot be equalized appropriately and system performance is degraded.

In this paper, a robust Kalman filter is proposed for estimating the channel in impuise noise environment. It uses an M-estimate [5] to identify and eliminate possible outliners which appear in the received signal. In addition, the recursive least Mestimate (RLM) algorithm proposed in [6] is used for estimating the AR parameters. Simulated results showed significant performance improvement over the conventional approach in estimation and equalization of fast fading channel in impulse noise environment.

\section{CHANNEL MODEL}

A model of discrete-time communications system with single-input single-output (SISO) is shown in Fig. I. Let $s(t)$ be the transmitted signal at time $t$ and $h(t, l)$ be the $l$ th tap of the impulse response of the time-varying channel. The received signal is given by:

$$
y(t)=\sum_{t=0}^{L-1} s(t-l) h(t, l)+w(t),
$$

where $w(t)$ is the zero-mean additive noise and $L$ is the number of channel taps. Normally $w(t)$ is assumed to be Gaussian distributed with variance $\sigma_{w}^{2}$. In practice, $w(t)$ might consist of impulse noise and it can be modeled as the contaminated Gaussian noise. The time-varying impulse response of the channel composes of a non-zero mean component $\bar{h}(l)$ and a zero-mean component $\tilde{h}(t, l)$ such that:

$$
h(t, l)=\bar{h}(l)+\tilde{h}(t, l) .
$$

To equalize this fast fading channel, it is usually modeled by an AR process [1]. More precisely, the time-varying component of the channel is formulated as:

$$
\tilde{\mathbf{h}}(t)=\mathbf{F}_{1} \tilde{\mathbf{h}}(t-1)+\cdots+\mathbf{F}_{M} \tilde{\mathbf{h}}(t-M)+\mathbf{u}(t),
$$

where $\mathrm{F}_{l}$ 's for $i=1, \ldots, M$ are the $\mathrm{AR}$ parameters and $\tilde{\mathbf{h}}(t)=[\tilde{h}(t, 0), \ldots, \tilde{h}(t, L-1)]^{T}$. The generating noise for the AR process $\mathbf{u}(t)=[u(t, l), \ldots, u(t, L-1)]^{T}$ is assumed to be a zeromean independent and identical distributed complex Gaussian random process with known covariance $\sigma_{u}^{2}$. Assume that the order of the AR process $M$ is known. Let $\mathbf{h}(t)=$ $\left[\tilde{\mathbf{h}}^{T}(t), \ldots, \tilde{\mathbf{h}}^{T}(t-M+1), \overline{\mathbf{h}}^{T}\right]^{T}$ be an augmented matrix of the channel impulse response, where the non-zero mean component vector is defined as $\overline{\mathbf{h}}=[\bar{h}(0), \ldots, \bar{h}(L-1)]^{T}$. Then fast fading channel can be written in the following state-space representation:

$\mathbf{h}(t)=\mathbf{A h}(t-1)+\mathbf{v}(t)$,
where $\mathbf{A}=\left[\begin{array}{ccccc}\mathbf{F}_{1} & \mathbf{F}_{2} & \cdots & \mathbf{F}_{M} & 0 \\ \mathbf{I} & 0 & \cdots & \mathbf{0} & 0 \\ \mathbf{0} & \ddots & & \vdots & \vdots \\ \vdots & & \mathbf{I} & \mathbf{0} & \mathbf{0} \\ \mathbf{0} & & \cdots & \mathbf{0} & \mathbf{I}\end{array}\right]$
and $\mathbf{v}(t)=[\mathbf{u}(t), \mathbf{0}, \ldots, 0]$.
$\mathbf{S i m i l a r l y ,}$ we define the input signal samples vector
$\mathbf{s}(t)=[s(t), \ldots, s(t-L+1)]^{T}$ and the observation matrix
$\mathbf{C}(t)=\left[\mathbf{s}^{T}(t), \mathbf{0}, \ldots, 0, \mathbf{s}^{T}(t)\right]$. From which, equation (1) can be
rewritten as:

Similarly, we define the input signal samples vector $\mathbf{s}(t)=[s(t), \ldots, s(t-L+1)]^{T}$ and the observation matrix rewritten as: 


$$
y(t)=\mathbf{C}(t) \mathbf{h}(t)+w(t) .
$$

From (4) and (7), if the AR parameters $F_{i}$ 's are known, a Kalman filter can be used to track the time-varying impulse response of the channel. In practice, however, the AR parameters are unknown or time-varying and thus online estimation is required. Usually, periodic training sequence is transmitted to the receiver to estimate the AR parameters. Efficient algorithms have been proposed to estimate the $A R$ parameters using LS algorithm [1]. Data symbols are then transmitted after the training period. At the receiver, each data symbol is detected using the current estimate of the channel. Once a data symbol is detected by, say, the decision feedback equalizer (DFE) or the Viterbi decoder, it is substituted into (7) for the estimation of next channel estimate by the Kalman filter. These two processes alternates until the next training period is reached, where the AR parameters will be estimated again. Coupled estimator with a Kalman filter running continuously in parallel with an AR parameters estimator was also proposed in [2]. Other techniques include the semi-blind approach in [4] where the training sequence is only transmitted once during initialization.

Unfortunately, both Kalman filter and LS algorithms are known to be sensitive to impulse noise. The accuracy of the channel and the AR parameters estimation can deteriorate by impulse noise coming from the communications channel, which leads to significant degradation of system performance. In this paper, we study a receiver that employs the robust Kalman filter and the RLM algorithm [6-8] to perform the robust estimation of the channel impulse response and AR parameters, respectively.

For simplicity, only SISO fading channel model is considered in this paper. However, the proposed architecture can be extended to MIMO fading channel models with minor modifications similar to [3] and [4].

\section{ROBUST AR PARAMETER ESTIMATION}

In order to estimate the AR parameters of the fast fading channel, the covariance matrices of the channel impulse response $\mathbf{R}_{\tilde{\mathbf{E b}}}(\tau)=E\left[\tilde{\mathbf{h}}(t+\tau) \tilde{\mathbf{h}}^{H}(t)\right]$ are first estimated. Consider the following conditional expectation [2]:

$$
\begin{aligned}
& E\left[[y(t+\tau)-\bar{y}(t+\tau)][y(t)-\bar{y}(t)]^{*} \mid \mathbf{s}(t)\right]= \\
& \boldsymbol{\varphi}^{H}(t, \tau) \theta(\tau)+\sigma_{w}^{2} \delta(\tau),
\end{aligned}
$$

where

$$
\begin{aligned}
& \bar{y}(t)=\sum_{t=0}^{L-1} s(t-l) \bar{h}(l), \\
& \varphi^{H}(t, \tau)=\left[s(t+\tau) s^{*}(t), \ldots, s(t+\tau) s^{*}(t-L+1),\right. \\
& \left.\ldots, s(t+\tau-L+1) s^{*}(t-L+1)\right], \\
& \theta(\tau)=\left[r_{\tilde{h}}(\tau, 0,0), \ldots, r_{\tilde{h}}(\tau, 0, L-1), \ldots, r_{\tilde{h}}(\tau, L-1, L-1)\right]^{T}, \\
& r_{\tilde{h}}(\tau, j, \eta)=E[\tilde{h}(t, j) \tilde{h} *(t+\tau, \eta)] .
\end{aligned}
$$

The zero-mean component of the channel estimate $\bar{h}$ is obtained from the robust Kalman filter during the channel estimation process. Equation (8) enables us to estimate $\theta(\tau)$, the channel tap covariance vector, from which we can estimate the AR parameters by solving the Yule-Walker equations in the AR parameters $F_{i}$ 's [9]. To proceed further, the expectations involved are approximated by their instantaneous values. That is:

$[y(t+\tau)-\bar{y}(t+\tau)][y(t)-\bar{y}(t)]^{*}=\varphi^{H}(t, \tau) \theta(\tau)+\sigma_{w}^{2} \delta(\tau)$.

The channel tap covariance vector $\theta(\tau)$ can be obtained recursively using the LS algorithm for different $\tau$.

Although the LS algorithm is very efficient in estimating the AR parameters, its performance can be degraded significantly in the presence of impulse noise [7]. In this paper, the RLM algorithm is used to minimize the effect of impulse noise to the AR parameters estimation.

The RLM algorithm minimizes an M-estimate-based objective function instead of the conventional LS objective function [7]:

$$
J_{\rho}(t)=\sum_{t=1}^{t} \lambda^{t-t} \rho(e(t)),
$$

where $\lambda$ is the forgetting factor and $\rho(\cdot)$ is a modified Huber $\mathrm{M}$-estimate function (MHF) given by [10]:

$$
\rho(e(t))= \begin{cases}e^{2}(\mathrm{t}) / 2 & \text { if }|e(t)|<\Delta \\ \Delta^{2} / 2 & \text { if }|e(t)| \geq \Delta\end{cases}
$$

The function $\rho(\cdot)$ is quadratic when the error $e(t)$ is smaller than the threshold $\Delta$. Otherwise, the function is a constant. Other M-estimate such as the Hampel three parts redescending function can also be used. Only MHF is considered in this paper because of its reasonable good performance and simplicity. It is apparent that the MHF is able to suppress outliners whenever $e(t)$ has abnormally large magnitude. The threshold $\Delta$ depends on the probability to detect the outliners and is estimated continuously.

The optimal solution $h(t)$ for minimizing $J_{\rho}(t)$ can be obtained by differentiating (14) with respect to $h(t)$ and setting the derivatives to zero. Its application to the estimation of the AR parameters using (9)-(13) is shown in Table 1. The RLM algorithm is similar to the RLS algorithm except for the weight function $q(\cdot)=\partial \rho(e) / \partial e$ which is given by:

$$
q(e(t))= \begin{cases}1 \text { if }|e(t)|<\Delta \\ 0 \text { if }|e(t)| \geq \Delta\end{cases}
$$

If $|e(t)|$ is great than $\Delta$, the Kalman gain vector $K(t)$ is equal to zero and the abnormal estimation error is prevented from entering the channel estimates. To estimate the threshold $\Delta$, for simplicity, $e(t)$ is assumed to consist of a Gaussian process that corresponds to the 'impulse-free' error signal and an impulsive component. Suppose the variance of the 'impulsefree' error signal $\hat{e}(t)$ is $\hat{\sigma}_{e}$, the probability of $|e(t)|>\Delta$ is [8]:

$$
\zeta_{\Delta}(t)=\operatorname{Pr}\{e(t) \mid>\Delta\}=1-e r f\left(\frac{\Delta}{\sqrt{2} \hat{\sigma}_{e}}\right)
$$

where $\operatorname{Pr}(\cdot)$ is the probability operator, $\operatorname{erf}(\cdot)$ is the error function and $\hat{\sigma}_{e}$ is the variance of $e(t)$. By choosing different values of $\zeta_{A}$, we have different confidence in detecting the presence of impulses. In this paper, $\zeta_{\Delta}$ is chosen as 0.01 and the 
corresponding threshold is $\Delta=2.576 \hat{\sigma}_{e}$. That means there is $99 \%$ confidence to reject the outliners when $|e(t)|>\Delta$.

The value of $\hat{\sigma}_{e}^{2}$ can be updated recursively by [6]:

$$
\begin{aligned}
& \hat{\sigma}_{e}^{2}=\lambda_{e} \hat{\sigma}_{e}^{2}+\left(1-\lambda_{e}\right) c_{1} \operatorname{med}(\Psi(t)), \\
& c_{1}=1.483\left(1+5 /\left(N_{w}-1\right)\right), \\
& \Psi(t)=\left|e^{2}(t), \ldots, e^{2}\left(t-N_{w}+1\right)\right| .
\end{aligned}
$$

Note that the median filter $\operatorname{med}(\cdot)$ is used to prevent the impulses from affecting $\hat{\sigma}_{e}^{2}$. The window size of the median filter is a compromise between the ability to response to sudden change of system transfer function and robustness to consecutive impulses. For a window length of $N_{w}$, the median filter is robust to $\left[N_{w} / 2\right]$ consecutive impulses. In practice, $N_{w}$ can be chosen between 5 to 11. A window length of 9 is chosen in this paper.

The convergence analysis of the RLM algorithm can be found in [10]. As we shall see later in the simulations section, the RLM algorithm is very effective in suppressing the adverse effect of the impulses, which otherwise might ruin the entire training process.

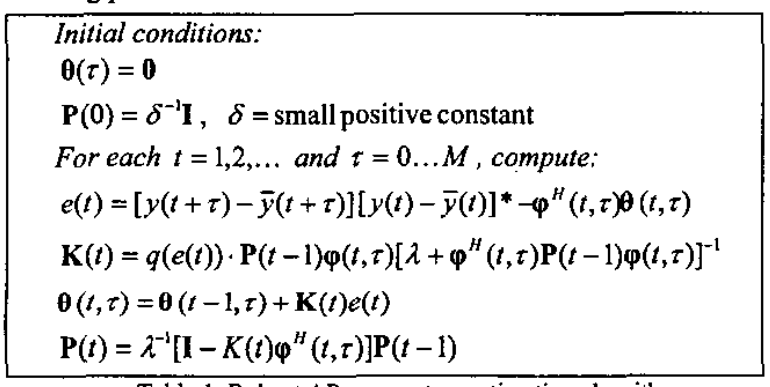

Table 1. Robust AR parameters estimation algorithm.

\section{ROBUST CHANNEL ESTIMATION}

Assume that the matrix $\mathbf{A}$ has been estimated by the RLM algorithm as described in section 3. A robust Kalman filter as shown in Table 2 can be used to estimate the channel response by means of the state-space model in (4)-(6). $\mathbf{C}(t)$ is derived from the decoded symbols during data transmission. The robust Kalman filter in Table 2 differs from the conventional Kalman filter in updating the Kalman gain:

$\mathbf{K}(t)=q(e(t)) \cdot \mathbf{P}(t \mid t-1) \mathbf{C}^{H}(t)\left[\mathbf{C}(t) \mathbf{P}(t \mid t-1) \mathbf{C}^{H}(t)+\sigma_{w}^{2} \mathbf{I}_{h}\right]^{-1}$,

where

$$
\mathbf{I}_{h}=\left[\begin{array}{cccc}
\mathbf{I} & \mathbf{0} & \cdots & \mathbf{0} \\
\mathbf{0} & \ddots & & \vdots \\
\vdots & & \mathbf{I} & \mathbf{0} \\
\mathbf{0} & \cdots & \mathbf{0} & \mathbf{0}
\end{array}\right]
$$

A weight function $q(\cdot)$ as defined in (16) is included and it serves the same purpose as the RLM algorithm to prevent the Kalman gain from being corrupted by impulses with abnormally large amplitude. The estimation of the threshold $\Delta$ is similar to the one described in section 3. Simulation results in section 6 showed that the proposed robust Kalman filter is less sensitive to impulse noise than its conventional counterpart. During the training period, the matrix $\mathbf{C}(t)$ is known. The estimated channel $\hat{\mathbf{h}}(t)$ is fed to the AR parameters estimator. After the training period, $\mathbf{C}(t)$ is replaced by its estimate $\hat{\mathbf{C}}(t)$, which is obtained from the DFE or Viterbi decoder.

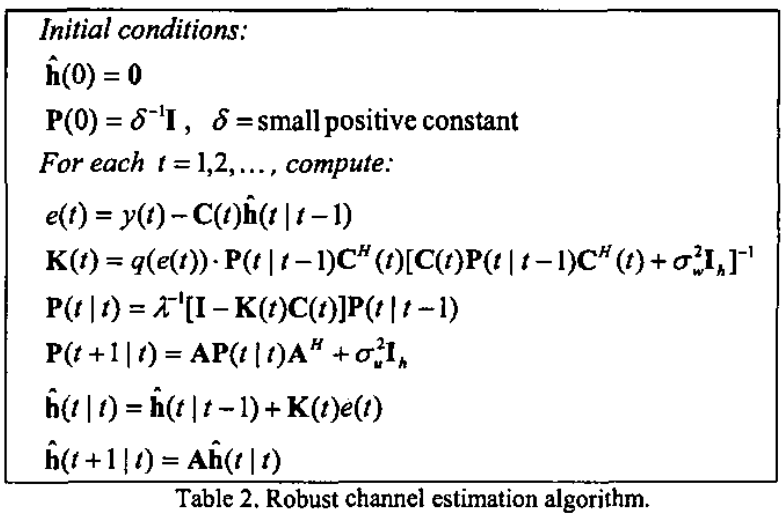

\section{THE PROPOSED ALGORITHM}

Without loss of generality, we only consider a semi-blind recursive coupled estimator in this paper. However, the proposed robust algorithms are also applicable to the periodic re-training cases.

During initialization, the training sequence is transmitted and thus the transmitted symbols $\mathbf{s}(t)$ are known. The robust Kalman filter and the RLM algorithm use the training sequence to estimate the channel and the AR parameters respectively. After the training period, the system operates in the blind mode. A DFE is used to detect the transmitted data symbol. The detected symbols $\hat{\mathbf{s}}(t)$ are then fed back to the coupled estimator to estimate the channel and AR parameters again. Fig. 2 shows the block diagram of the channel estimation and equalization processes. The proposed algorithms are summarized as follow.

\subsection{Training mode}

Step 1: Generate the matrix $\mathbf{C}(t)$ from the known training symbols.

Step 2: Estimate the channel and the AR parameters using the proposed robust Kalman filter and RLM algorithms.

\subsection{Blind mode}

Step 1: Decode the received signal using DFE to obtain the decoded data symbols $\hat{\mathbf{C}}(t)$.

Step 2: Run the robust Kalman filter and AR parameters estimator. Update the channel using the decoded data symbols.

\section{SIMULATIONS}

Simulation under impulse noise environment is performed to evaluate the performance of the proposed robust channel estimation and equalization algorithms. The performance of the proposed robust algorithms is compared to the conventional Kalman filter and LS algorithm. In our experiment, the results are obtained by averaging over 100 independent runs. A fading channel with length $L=2$ is considered, which is modeled as an AR process with order $M=1$ and with mean impulse response $\overline{\mathbf{h}}=[1+0.2 j,-0.5+0.5 j]^{T}$. 
The true $A R$ parameters matrix $F_{1}$ is:

$$
\mathbf{F}_{1}=\left[\begin{array}{cc}
0.3 & 0.8 \\
-0.5 & 0.3
\end{array}\right]
$$

and $\sigma_{y}^{2}$ is chosen as 0.005 . The input symbols $s(t)$ are drawn from a 16-QAM constellation with $\sigma_{w}^{2}=10^{-2}$. An initial training period of 1000 samples is used. Both the conventional and robust receivers start to decode the data after the training period.

To visualize more clearly the effects of the impulses, a chain of impulses is generated using a Gaussian distribution of $\sigma_{i}^{2}=200$ (i.e. a contaminated Gaussian noise modeled with the position of the impulses fixed for clarity of presentation.) and are placed at $t=1700,1810,1920,2030,2130$ as shown in Fig. 3. $\lambda$ and $\lambda_{e}$ are chosen as 0.99 and 0.9 , respectively. Fig. 4 shows the recursive estimation of the real part of $F_{1}(1,1)$, where $\mathbf{F}_{1}(i, j)$ is the $(i, j)$ th component of matrix $\mathbf{F}_{1}$. When there are impulses, the performance of the conventional algorithms is affected significantly, while the performance of the proposed robust algorithms is not so affected by the impulses. Fig. 5 shows the recursive estimation of the real part of $\bar{h}(0)$. The effect of impulses on the performance of the proposed algorithm is minimized while the performance of the conventional algorithms is affected to a greater extent.

\section{CONCULSION}

The effect of impulse noise in the estimation and equalization of fast fading channel is studied. A new robust Kalman filter and the RLM algorithm are proposed to estimate the channel and AR parameters in impulse noise environment. Simulations showed that the proposed algorithms perform better than the conventional algorithms under the stated conditions.

\section{REFERENCES}

[1] M. K. Tsatsanis, G. B. Giannakis and G. Zhou, "Estimation and Equalization of Fading Channels with Random Coefficients," Signal Processing, vol. 53, pp. 211-229, Sep 1996.

[2] L. M. Davis, I B. Collings and R. J. Evans, "Identification of Timevarying Linear Channels," in Proc. ICASSP'97, Munich, Germany, Apr. 1997.

[3] C. Komninakis, C. Fragoulia, A. H. Sayed and R. D. Wesel, "Adaptive Multi-input Multi-output Fading Channel Equalization using Kalman Estimation," in Proc. ICC'2000, New Orleans, LA, Jun. 2000.

[4] M. Enescu, M. Sirbu and V. Koivunen, "Recursive Semi-blind Equalizer for Time-varying MIMO Channels," in Proc.Workshop on SSP, Singapore, Aug. 2001.

[5] S. R. Kim and A. Efron, "Adaptive robust impulse noise filtering," IEEE Trans. Signal Processing, vol. 43, Aug 1995.

[6] Y. Zou, S. C. Chan and T. S. Ng, "A recursive least M-estimate (RLM) adaptive filter for robust filtering in impulse noise," IEEE Signal Processing Letter, vol. 7, pp. 324-326, Nov 2000.

[7] Y. Zou, S. C. Chan and T. S. Ng, "A Robust M-estimate Adaptive Filter For Impulse Noise Suppression," in Proc. ICASSP'99, Phoenix, AZ, Mar. 1999.

[8] Y. Zou, S. C. Chan and T. S. Ng, "Robust M-estimate adaptive filtering," IEE Proc. Vision, Image and Signal Processing, vol. 148, pp. 289-294, Aug 2001.

[9] S. Haykin, Adaptive Filter Theory, New Jersey: Prentice Hall, 1996.

[10] Y. Zou, PhD Thesis, The University of Hong Kong, 2000.

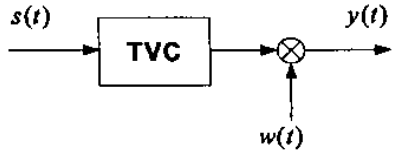

Fig. 1. Fading channel model.

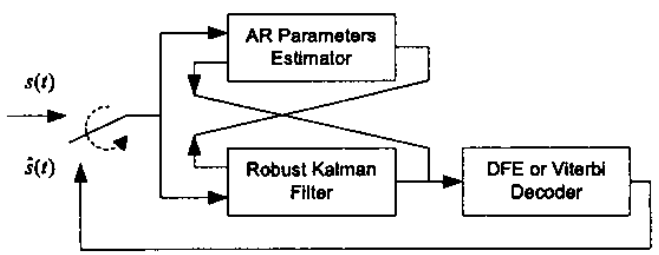

Fig. 2. The proposed coupled estimator consisting of a robust Kalman filter and an AR parameters estimator using the RLM algorithm.

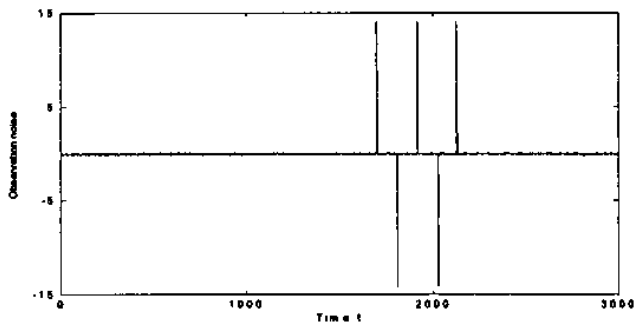

Fig. 3. Observation noise in the received signal.

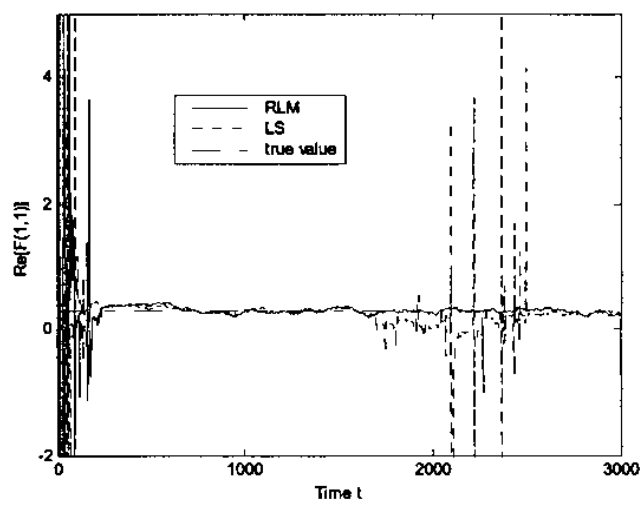

Fig. 4. Estimation of the real part of $\mathbf{F}(1,1)$ under impulse noise.

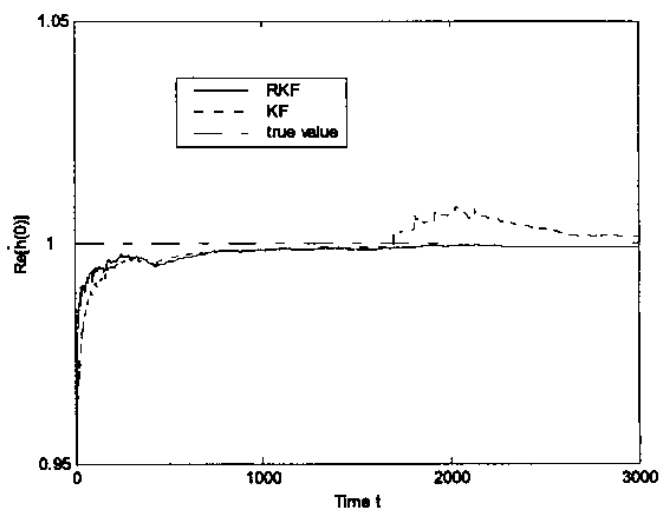

Fig. 5. Estimation of the real part of $\bar{h}(0)$ under impulse noise. KF: conventional Kalman filter. RKF: robust Kalman filter. 